\title{
Spaceborne SAR product geolocation accuracy: A Sentinel-1 update
}

Schubert, Adrian ; Small, David ; Meier, Erich ; Miranda, Nuno ; Geudtner, Dirk

\begin{abstract}
Sentinel-1A (S1A), launched by the European Space Agency (ESA) on April 3, 2014, is a state-of-the-art spaceborne Synthetic Aperture Radar (SAR) Earth observation platform. S1A products are expected to provide high and consistent geolocation accuracy. As of the end of May, 2014, the satellite has not yet reached its reference orbit. However, estimation of product geolocation accuracy is ongoing, and improvements are continually being made. The results reported here represent the early situation, with continued progress expected.
\end{abstract}

DOI: https://doi.org/10.1109/IGARSS.2014.6947025

Posted at the Zurich Open Repository and Archive, University of Zurich

ZORA URL: https://doi.org/10.5167/uzh-101575

Conference or Workshop Item

Published Version

Originally published at:

Schubert, Adrian; Small, David; Meier, Erich; Miranda, Nuno; Geudtner, Dirk (2014). Spaceborne SAR product geolocation accuracy: A Sentinel-1 update. In: Geoscience and Remote Sensing Symposium (IGARSS), 2014 IEEE International, Québec (Canada), 13 July 2014 - 18 July 2014. IEEE Xplore, 2675-2678.

DOI: https://doi.org/10.1109/IGARSS.2014.6947025 


\title{
SPACEBORNE SAR PRODUCT GEOLOCATION ACCURACY: A SENTINEL-1 UPDATE Adrian Schubert ${ }^{1}$, David Small ${ }^{1}$, Erich Meier ${ }^{1}$, Nuno Miranda ${ }^{2}$, Dirk Geudtner ${ }^{3}$
}

\author{
(1) Remote Sensing Laboratories, University of Zurich, Switzerland \\ (2) European Space Agency ESA-ESRIN, Frascati, Italy \\ (3) European Space Agency ESA-ESTEC, Noordwijk, The Netherlands
}

\begin{abstract}
Sentinel-1A (S1A), launched by the European Space Agency (ESA) on April 3, 2014, is a state-of-the-art spaceborne Synthetic Aperture Radar (SAR) Earth observation platform. S1A products are expected to provide high and consistent geolocation accuracy. As of the end of May, 2014, the satellite has not yet reached its reference orbit. However, estimation of product geolocation accuracy is ongoing, and improvements are continually being made. The results reported here represent the early situation, with continued progress expected.
\end{abstract}

Index Terms - Sentinel-1A, geolocation accuracy, SAR calibration/validation

\section{INTRODUCTION}

Operating in C-band, S1A's stripmap products achieve spatial resolutions better than $5 \mathrm{~m}$ (Table 5-1 in [1]). Especially in light of the increasing need for SAR timeseries and combination of S1A data with other data sources, S1A products are expected to provide equally high and consistent geolocation accuracy. The S1A ground segment includes several groups providing scientific expertise and engineering support during the mission's commissioning phase [4]. As one of these groups, the SAR team at the Remote Sensing Laboratories, University of Zurich, Switzerland is responsible for the S1A product geometric calibration and validation. At the time of this writing (end of May, 2014), S1A has not yet reached its final reference orbit; this will be achieved within 1-2 months. Early calibration issues and ongoing orbital manoeuvres have presented a challenge to the generation of SAR products with consistently high geolocation accuracy. However, in spite of these challenges, product geolocation estimates have been improving steadily.

\section{METHODS}

The estimation of SAR product geolocation accuracy involves the empirical determination of the system-inherent range and azimuth timing biases. Usually, these biases are estimated using point targets with accurately surveyed positions, such as corner reflectors (CRs) or active transponders. Their cartographic positions are transformed into slant-range image coordinates using range-Doppler geolocation [3]. It is important to keep in mind that the prediction accuracy is critically dependent on the annotated range and azimuth timing information and the quality of the orbital state vectors. It is, in effect, these parameters that reflect the inherent geolocation accuracy of the imaging system.

The timing parameters consist of known and unknown components. The system-inherent biases are now better understood than in the days following the launch; the unknown components will soon be better characterised through the external calibration exercises.

Satellite positions are recorded during acquisition and can be integrated into the products. The GNSS positions are later refined and delivered as higher quality restituted or precise orbit files by the Precise Orbit Determination (POD) service.

The predicted $\mathrm{CR}$ coordinates in a given image can be compared with the actual imaged intensity peak locations. The resulting offset will be the sum of the systeminherent biases and state vector errors mentioned above, in addition to perturbing effects such as the atmospheric path delay (PD), plate tectonics and the solid Earth tide (SET) [6][7].

In the era of Sentinel-1, with improved spatial resolution and greater localisation accuracy requirements, these additional influences need to be modelled and separated from the overall biases in order to determine the true system-inherent biases. An overview of the methods used to mitigate these influences is given in [6].

Given that S1A is being manoevred toward its reference orbit at the time of this writing, there are at present not enough products available with stable properties over the calibration test sites to make reliable bias estimation feasible. As a result, PD and SET modelling and mitigation were not yet performed for the S1A products received so far.

S1A has a two-dimensional pixel location accuracy specification of $2.5 \mathrm{~m}$ for its stripmap mode products (section 5.5.2.1 in [2]). As part of the calibration/validation team, we will aim to achieve this goal once S1A reaches its reference orbit. To date, work was aimed at gaining consistency and an improved understanding of the influences on the product quality. Based on recent 


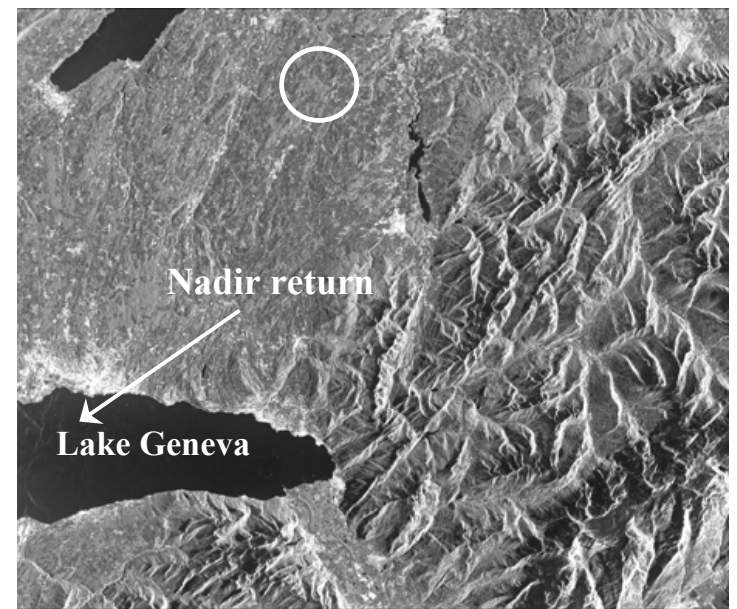

Fig. 1 Sentinel-1 stripmap product from beam S6 over western Switzerland from a descending orbit, acquired May 4, 2014. Test site Torny-le-Grand with corner reflectors is circled; HH polarisation is shown.

measurements, this consistency has nearly been achieved, in spite of the continuing orbital manoeuvres. Using image products acquired mainly in stripmap mode over CRs and transponders, range and azimuth image location predictions are generated for each target and compared with the measured imaged target locations. The measured offsets can be converted to timing biases, which can be considered to achieve a better calibrated system. Subsequent products generated with updated processors can benefit from the calibration measurements.

\section{STRIPMAP PRODUCT OVERVIEW}

S1A stripmap products were acquired over our test site in the west of Switzerland, Torny-le-Grand. Four trihedral CRs similar to those used in [7] were set up there and their positions measured to $\sim \mathrm{cm}$ accuracy using differential GPS (DGPS). A recent acquisition that included the site is shown in Fig. 1; the site's location is indicated with the white circle. Two of the CRs are oriented towards the descending orbit track; the other two face the ascending orbit. This pairwise setup not only provides two estimates of geolocation accuracy per product; the consistency of offset measurements between the two also supports the plausibility of the surveyed positions.

Several active transponders in Europe and Canada also serve as calibration targets for S1A. One of these, the NLR transponder, is located in the Netherlands and is visible at the centre of the stripmap product extract shown in Fig. 2.

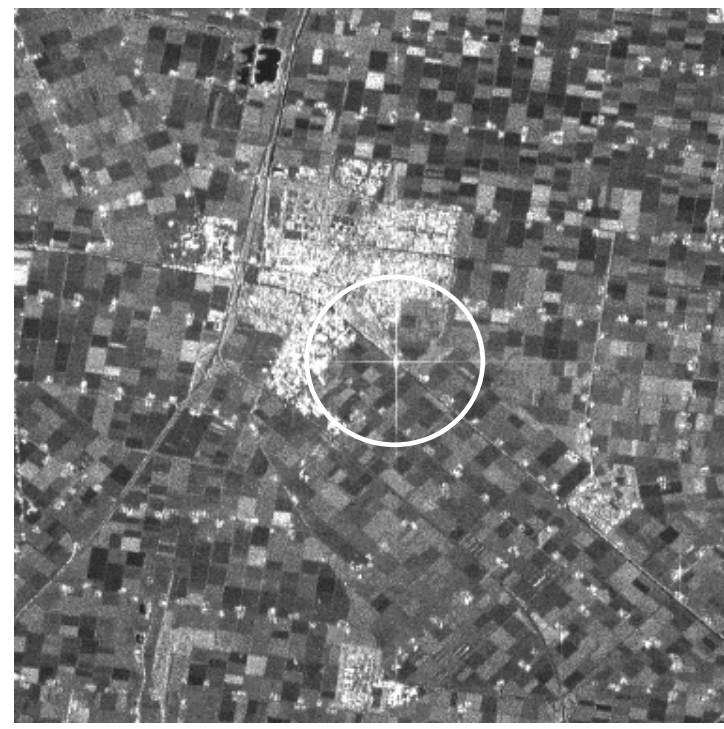

Fig. 2 Extract of a Sentinel-1 beam S6 stripmap product over the Netherlands containing the NLR transponder (circled). Acquired from a descending orbit April 19, 2014.

\section{SENTINEL-1A: CURRENT STATUS}

At the time of this writing, S1A is in an extended orbit acquisition phase (OAP), being nudged towards its reference orbit, which should be achieved within 1-2 months. Despite the unforeseen challenges of the extended OAP, satisfying results are now beginning to be achieved, as Fig. 3 and Fig. 4 illustrate. The beam S6 (incident angle $\sim 45^{\circ}$ ) stripmap images of the corner reflectors deployed at Tornyle-Grand are shown, with yellow crosses centred on their predicted locations. $3 \times 3$ oversampled imagettes are at the bottom; 1 x 1 indicates no oversampling was done.

Fig. 3 shows two products generated from the same acquisition. At the top is the slant range complex image (SLC, amplitude shown), at the bottom is the full-resolution ground range detected (GRDF) product. Compared with an SLC product, the generation of geolocation predictions for a GRDF includes an additional ground-to-slant range conversion, performed using an annotated polynomial. The visibly similar predictions for the geolocation predictions in the SLC and GRDF cases attest to the high quality of the polynomial.

Also visible in Fig. 3 (top and bottom) is the clear offset between the predicted and imaged CR locations in the range direction. When new products are generated, the nominal chirp replica is used for processing. Ideally, the mean time delay from previous replicas derived from the internal calibration is applied to the nominal replica (see [5] and Tables 6-52 and 6-53 in [8]). However, in Fig. 3 the replica delay term was not applied during processing, effectively shifting the predicted target range positions. 

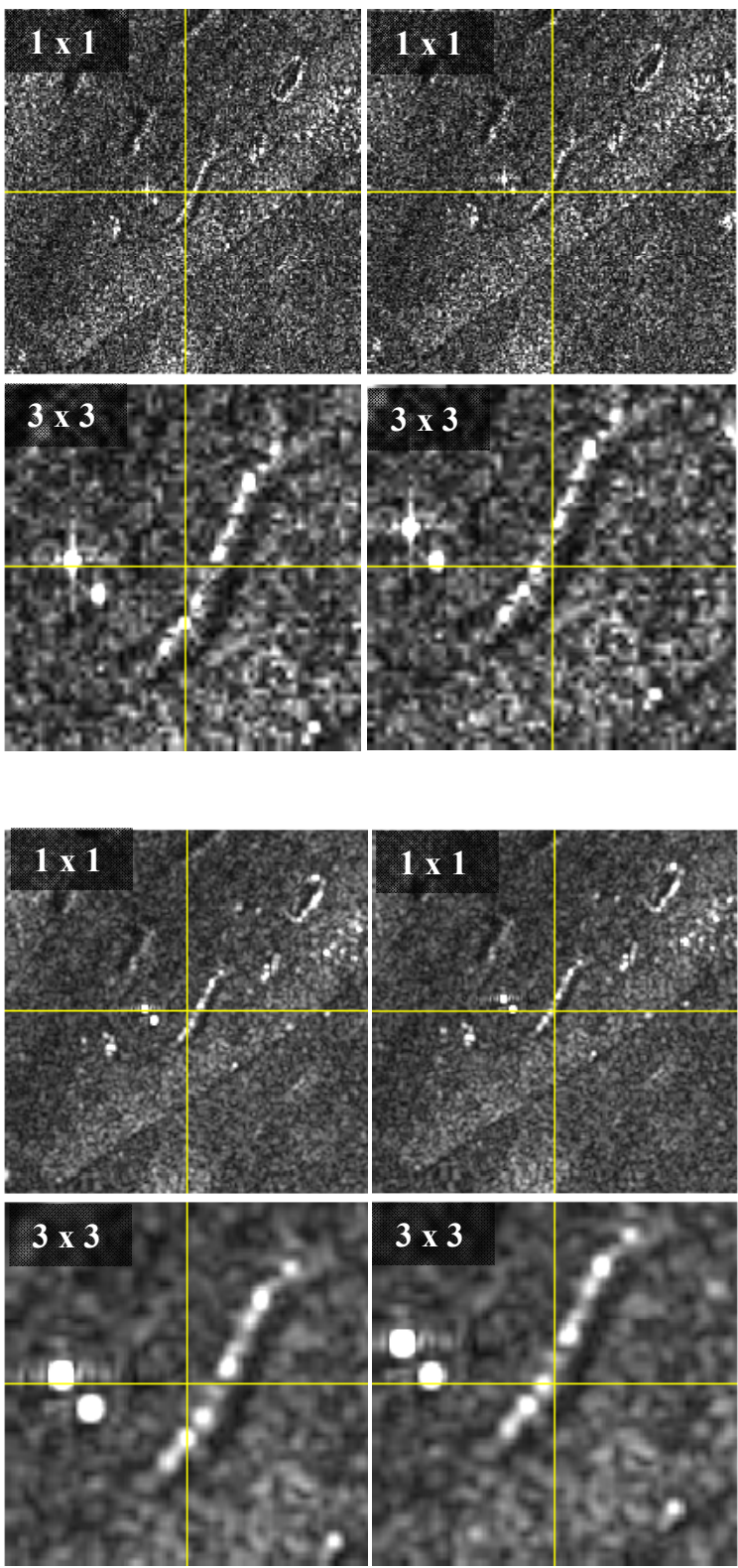

Fig. 3 Sentinel-1 stripmap image extracts of Torny-leGrand corner reflectors. Yellow crosses indicate their predicted positions (based on restituted orbit file and nominal chirp replica with an initially incorrect offset annotation). Acquired May 4, 2014 using beam S6 (see Fig. 1). Top: SLC product; Bottom: GRDF product.

The processor was subsequently adjusted to correctly apply the chirp replica delay, as can be seen in Fig. 4, where the range offset that was visible in Fig. 3 has disappeared. The predictions were generated using the precise orbit files (AUX_POEORB). Additionally, the surveyed $\mathrm{CR}$ coordinates were transformed into the international terrestrial reference frame ITRF2008, by modelling tectonic plate movement. The tectonic drift causes the European reference frames (including the Swiss frame we used, CHTRF95) to drift away from the global frame, introducing artificial offsets if not properly taken into account [6].

The mean range offset measured for this product was -0.63 samples $(-2.0 \mathrm{~m})$; in azimuth the offset was 0.89 samples $(3.6 \mathrm{~m})$. This represents the best estimate of S1A's geolocation accuracy to date. It should not be forgotten that atmospheric path delay (PD) was not yet considered when generating these estimates. PD modelling will lengthen the predicted range and increase the current $2.0 \mathrm{~m}$ bias by between $\sim 2$ and $4 \mathrm{~m}$, so the current range error cannot be seen as final.

The NLR transponder north of Flevoland is visible in the image extract shown in Fig. 5. The product was focused used the transponder's delay term, optimising the azimuth reference function and hence image quality for the transponder itself. For this prediction, the state vectors downlinked at acquisition time were used. The resulting offsets were found to be -2.38 samples $(-7.6 \mathrm{~m})$ in range and 1.29 samples $(5.2 \mathrm{~m})$ in azimuth. A later estimate was made using a more accurate precise orbit file generated ad hoc by the POD service, which is also under commissioning and not yet operational. The new orbit file improved the estimates slightly: -2.10 samples $(-6.7 \mathrm{~m})$ in range, 0.79 samples $(3.2 \mathrm{~m})$ in azimuth. While tectonic-compensated ITRF2008 reference positions were used for the transponder, the results are not "final," as PD modelling was not yet applied.

\section{CONCLUSION}

In spite of a delay in attaining its final orbit, Sentinel-1A is already demonstrating good geolocation accuracy, especially with the higher quality restituted and precise orbit files generally available several days following product generation. Azimuth offsets of $\sim 1-2$ samples are observed at the moment. Refinement of range predictions will be tested in the near future on a diverse set of products acquired in multiple modes over different test sites. This will lead to the generation of updated processor calibration constants.

Iterative improvements are expected for the coming weeks. The final stage of the calibration/validation phase will include systematic accounting for PD, SET and tectonic effects as well as all known system-inherent biases. The measurements over calibration targets will be reviewed and fed back into the processor; the result will be a wellcalibrated system delivering consistently high geolocation quality. 

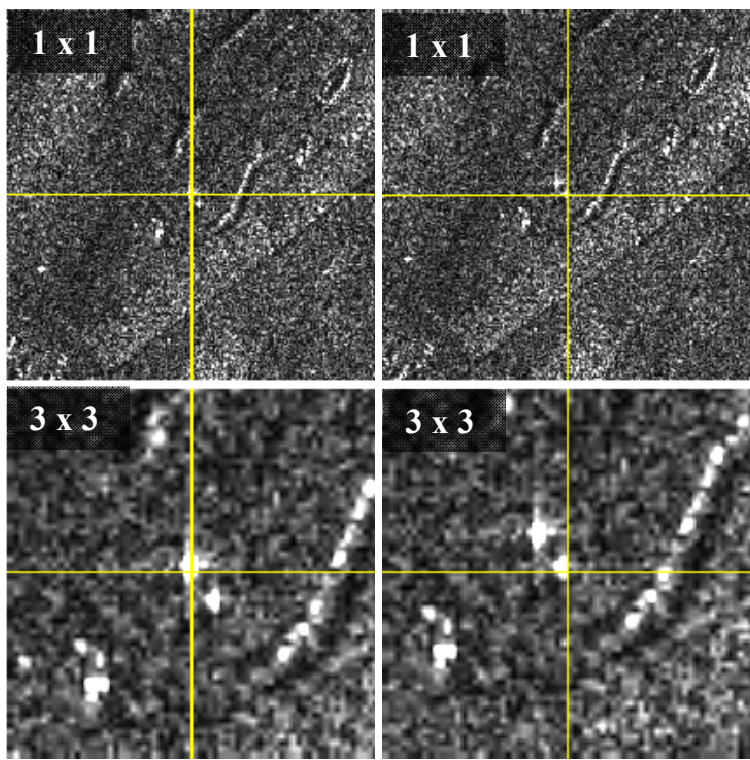

Fig. 4 Sentinel-1 stripmap image extract of Torny-leGrand corner reflectors. Yellow crosses indicate their predicted positions (based on the precise state vector file). Acquired May 4, 2014 using beam S6 (see Fig. 1).

\section{REFERENCES}

[1] M. Aulard-Macler, "Sentinel-1 Product Definition," Iss.2/Rev.4, MDA technical note Ref. S1-RS-MDA-52-7440, 126 p., 2012.

[2] GMES Sentinel-1 Team, "GMES Sentinel-1 System Requirements Document," Iss. 3/Rev. 3, ESA-ESTEC technical note Ref. S1 RS ESA SY 0001, 143p., 2010.

[3] E. Meier, U. Frei and D. Nüesch, "Precise terrain corrected geocoded images," Chap 7. In: Schreier G (ed) "SAR geocoding: data and systems," Herbert Wichmann Verlag GmbH, Karlsruhe, Germany, 1993.

[4] P. Potin, "Sentinel-1 User Handbook," Rev. 0 (draft), European Space Agency technical note, 80p., 2013.

[5] E. Schied, "SAR Instrument Calibration and Charaterization Plan", EADS astrium technical note Ref. S1-PL-ASD-PL-0001, Iss. 6/Rev. 1, 76 p., February 2, 2012

[6] A. Schubert, M. Jehle, D. Small and E. Meier, "Mitigation of atmospheric perturbations and solid Earth movements in a TerraSAR-X time-series," Journal of Geodesy, vol. 86, no. 4, pp. 257-270, 2012.

[7] A. Schubert, M. Jehle, D. Small and E. Meier, "COSMOSKYMED, TerraSAR-X, and RADARSAT-2 Geolocation Accuracy after Compensation for Earth-System Effects", Proc. of IGARSS'12, Munich, Germany, pp. 3301-3304, 2012.
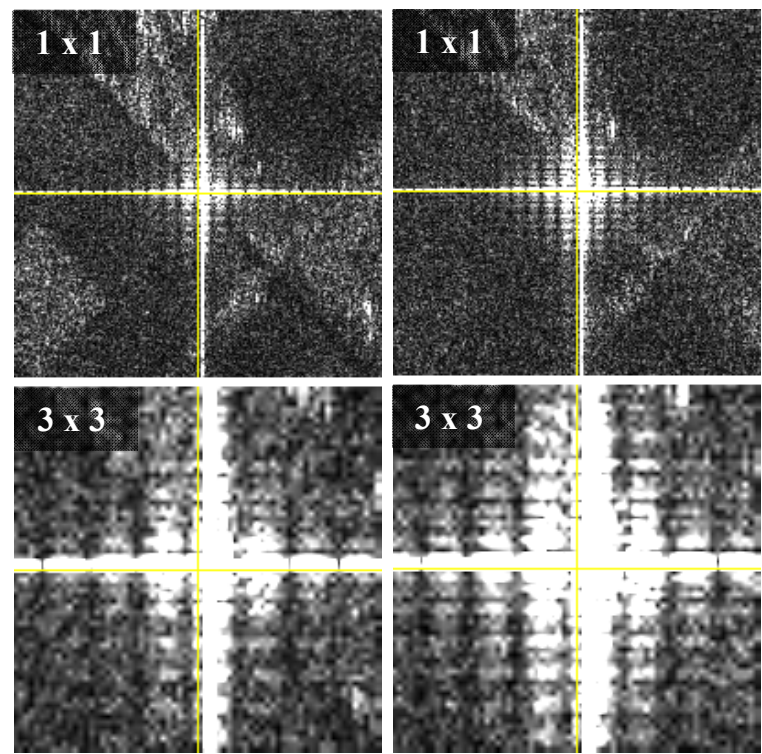

Fig. 5 Sentinel-1 geolocation prediction over imagettes of the NLR transponder in the Netherlands product acquired April 19, 2014. The predictions are based on the state vectors recorded during the acquisition.

[8] C. Thain, "Sentinel-1 Product Specification", MDA Document Number: SEN-RS-52-7441, Iss.2/Rev.7, 177 p., January 30, 2013. 